\title{
Laparoscopic Pyeloplasty for Ureteropelvic Junctions Obstruction in Adults: 6 Years' Experience in One Center
}

\author{
Rikke Søgaard Tolstrup, Marie Thue Pank, Lotte Sander, and Torben Dørflinger \\ Department of Urology, Aalborg University Hospital, Aalborg, Denmark \\ Correspondence should be addressed to Rikke Søgaard Tolstrup; r.tolstrup@rn.dk
}

Received 14 October 2016; Revised 7 March 2017; Accepted 26 April 2017; Published 11 June 2017

Academic Editor: Christian Schwentner

Copyright (C) 2017 Rikke Søgaard Tolstrup et al. This is an open access article distributed under the Creative Commons Attribution License, which permits unrestricted use, distribution, and reproduction in any medium, provided the original work is properly cited.

Objective. Ureteropelvic junction obstruction (UPJO) is a common cause of symptomatic ureteral obstruction. The aim of this study is to assess the outcome of laparoscopic pyeloplasty in patients with UPJO. Patients and Methods. Medical reports of 47 UPJO patients treated with laparoscopic pyeloplasty were retrospectively analysed. All patients were recruited from our center in the period 2004-2011. Results. We evaluated 47 patients. Mean age was 36 years and mean hospital stay 3.6 days. 42 (79\%) of the patients had pain and 46 (98\%) were diagnosed with hydronephrosis. 19 patients (40\%) had a renal function below $40 \%$ of the affected kidney and $49 \%$ had impaired renal scan drainage. Postoperative significant improvement in pain score and renal scan drainage was found in $92 \%$ and $47 \%$ of the patients, respectively. Improvement of renal function $>10 \%$ was found in 11 patients (23\%); the function remained stable in 31 patients (66\%) and deteriorated $>10 \%$ in 5 patients (11\%). We found no correlation between sex or age and the outcome. Conclusion. Laparoscopic pyeloplasty for UPJO leads to relief of pain and preserved or improved renal function in the majority of the patients. Overall laparoscopic pyeloplasty is an efficient treatment for UPJO.

\section{Introduction}

Ureteropelvic junction obstruction (UPJO) either idiopathic, iatrogenic, or due to compression of aberrant vessels is a common urological problem. Untreated the disease can cause renal failure, urinary tract infection, urolithiasis, and other symptoms as pain [1].

The primary gold of intervention is to preserve or improve renal function and relieve symptoms. Surgical reconstruction is the golden standard for treatment and there are several options for surgical intervention [2].

1.1. Endourologic Management. Today the endourologic management is mostly performed as a retrograde ureteroscopic endopyelotomy. However, the procedure may be performed as percutaneous antegrade one either as a endopyelotomy or endopyeloplasty.

The procedure is performed with a lateral incision through the obstructing proximal ureter using a cold knife or the holmium laser. Alternatively the obstruction can be dilated with a cautery wire balloon [3].
1.2. Pyeloplasty. This surgical management can be performed as open surgery, laparoscopic or robot-assisted. The technique is performed by dissecting the part of the ureter and renal pelvis with the obstruction, then spatulating the ureter, and making an anastomosis to the renal pelvis.

Laparoscopic standard approach is transperitoneal, but the procedure can also be performed by an retroperitoneal approach, anterior extraperitoneal approach, laparoendoscopic single-site surgery approach, or robotic-assisted approach. The most widely used method is the AndersonHynes pyeloplasty or one of the nondismembered methods such as Foley Y-V plasty. Methods like Culp-DeWeerd spiral flap pyeloplasty, Scardino-Prince Vertical Flap, Davis intubated ureterotomy, and ureterocalicostomy can also be used [3]. Figure 1 shows the laparoscopic approach.

1.3. Nephrectomy. This treatment option is rarely the procedure of choice and is only used when the affected kidney is nonfunctioning, the patient has symptoms, and other treatment options are not preferred [3]. 


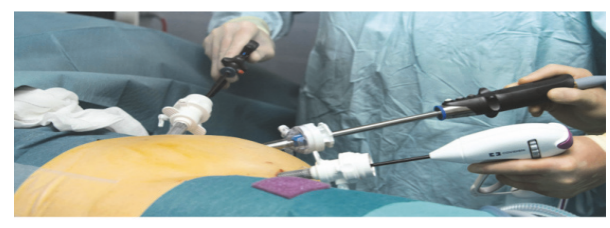

FIGURE 1: Laparoscopic pyeloplasty

Pyeloplasty is currently the standard treatment for most cases of UPJO. The success rate with endourologic techniques is lower and has proven not to be comparable with those of pyeloplasty.

Traditionally, the surgical procedure was performed as an open pyeloplasty, but since the development of minimal invasive surgical techniques, the standard is now to perform the procedure laparoscopically.

The European Association of Urology recommended in their guidelines that standard option of treatment should be pyeloplasty performed by a laparoscopic approach [4].

In 2015 a total of 120 pyeloplasty cases, primary as minimally invasive surgery, were performed in Denmark.

The aim of this study is to assess the subjective symptoms and objective outcome in patients with UPJO undergoing a laparoscopic pyeloplasty in our center.

\section{Patients and Methods}

The study was performed as a retrospective analysis of medical reports of patients with UPJO treated with laparoscopic pyeloplasty, using the Anderson-Hynes dismembered technique or a nondismembered technique as a Y-V or flapplasty. All medical reports were collected from our center: The Department of Urology, Aalborg University Hospital, between 2004 and 2011.

All patients with UPJO were evaluated, and only patients who had undergone pyeloplasty $(n=56)$ were included in this study. Patients that had open surgery were excluded, resulting in a total of 47 patients.

The included patients all had UPJO, which was mainly diagnosed by subjective symptoms such as flank pain, chronic urinary tract infection, and urolithiasis and the diagnosis was confirmed by intravenous ureterography.

Indications for surgery were symptomatic UPJO such as flank pain, urinary tract infection, impaired renal function, and/or decline in renal function over time monitored on a diuretic renal scan.

All patients who preoperatively had symptoms underwent surgery with cystoscopy insertion of a double-J stent. If the stent showed a beneficial effect on the symptoms, the patients were considered for operation.

All the patients had laparoscopic surgery with pyeloplasty either as conventional laparoscopy or as a robot-assisted procedure. The surgical technique of choice was surgeon dependent; however in general all patients with aberrant vessel had the pyeloplasty by the Anderson-Hynes technique. The remaining patients with no aberrant vessels had either Anderson-Hynes, Y-V, or flap-plasty, depending on size and ethology of the obstruction and the surgeon.
TABLE 1: Characteristics of patients with UPJO.

\begin{tabular}{lc}
\hline Characteristic & $\begin{array}{c}\text { Number } \\
\text { (range) }\end{array}$ \\
\hline Number of patients & 47 \\
$\quad$ (i) Male & 23 \\
$\quad$ (ii) Female & 24 \\
Age, yr.: mean (range) & $36(15-73)$ \\
$\quad$ (i) Male & $32(15-68)$ \\
$\quad$ (ii) Female & $40(18-73)$ \\
Days in hospital: mean \\
(range) \\
$\quad$ (i) Male & $3,6(2-8)$ \\
(ii) Female & $3,7(2-8)$ \\
\hline
\end{tabular}

During surgery a double-J stent was routinely inserted in all patients; the stent was removed at follow-up.

At follow-up the patients' subjective symptoms were evaluated as well as the objective outcome, which were monitored by ureterography and renal scans.

\section{Results}

The medical reports of a total of 56 patients who underwent pyeloplasty were examined; 9 patients were excluded because they had open procedure, leaving 47 patients available for evaluation, 24 females and 23 males. All 47 patients were characterised by gender, age, and mean hospital stay (Table 1 ). At surgery, the patient's age ranged from 15 to 73 years; mean age was 36 years.

The mean hospital stay was 3.6 days, ranging from 2 to 8 days.

In order to assess the outcome after pyeloplasty, postoperative symptoms and objective findings were identified (Figure 2).

$79 \%$ of the patients had subjective symptoms before operation dominated by flank pain. $98 \%$ patients were diagnosed with hydronephrosis in preoperative imaging. $64 \%$ of the patients had renal function blow $45 \%$ of the affected kidney at renography, while a function below $40 \%$ was seen in $40 \%$ of the patients. Impaired renal scan drainage was found in $49 \%$ of the patients.

Mean days until follow-up were 45,5 days with a range from 18 to 151 days.

The results after operation are presented in Figure 3.

Postoperative significant improvement in pain score and renal scan drainage was found in $92 \%$ and $47 \%$ of the patients, respectively. Postoperative imaging showed improvement in $65 \%$ of the patients.

Improved renal function more than $10 \%$ was seen in 11 patients (23\%); the function remained stable in 31 patients (66\%) and deteriorated $>10 \%$ in 5 patients $(11 \%)$.

We found no correlation between sex or age and the outcome of the operation.

One patient required repeat pyeloplasty due to reobstruction. Furthermore one patient underwent a reoperation due to severe postoperative pain. 


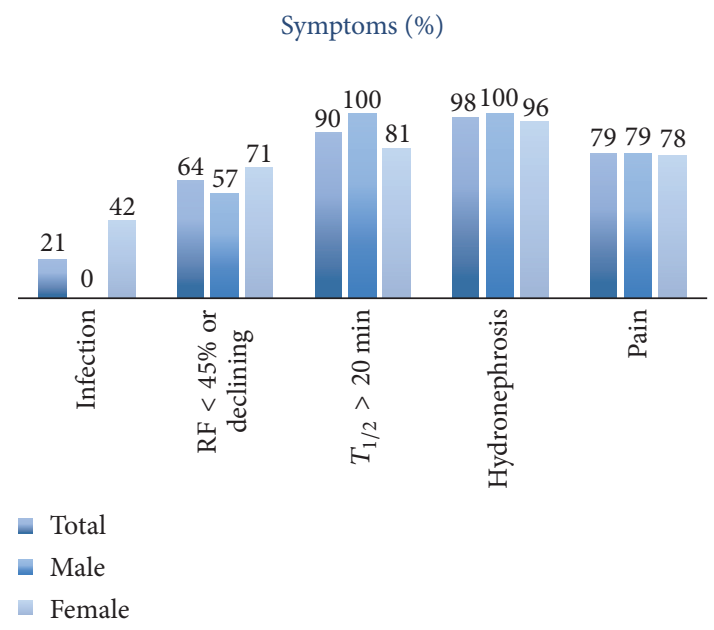

Figure 2: Preoperative symptoms and objective findings.

\section{Discussion}

In this study we show that laparoscopic pyeloplasty is an efficient treatment for UPJO, with a significant improvement in pain and renal scan drainage in $92 \%$ and $47 \%$ of the patients, respectively.

$89 \%$ of the patients had stabilized their renal function or even improved it with more than $10 \%$. The result must be taken into consideration that the study population was quite small with only 47 patients.

This study was performed as a retrospective analysis based on medical reports, which has its limitations. There were no standardizations on subjective and objective symptoms. The patients did not follow a standard programme for presurgery examination or postoperative follow-up.

The use of validated questionnaires for assessment of subjective symptoms, as pain, would have strengthened our study.

The urography was performed and described by different radiologists and there was no standard or measurement for the degree of hydronephrosis.

In our study population there was no differentiation between patients who had conventional laparoscopic procedure or robot-assisted one (Da Vinci robot system). Furthermore there was no differentiation in the etiology causing the UPJO and the surgical technique used.

Our results are equal to a recent meta-analysis by Wang et al. who shows an overall effect on laparoscopic pyeloplasty on $88 \%$ of the patients [5].

Recent studies have shown similar results and no statistical significant difference in overall success rates comparing traditionally open surgery technique with minimal invasive surgery.

Several studies have demonstrated that adults with UPJO treated with pyeloplasty as minimally invasive surgery have a lower risk of complications, transfusions, prolonged hospital stay, and cosmetic outcome and a general lower morbidity compared to patients that had open surgery $[6,7]$.

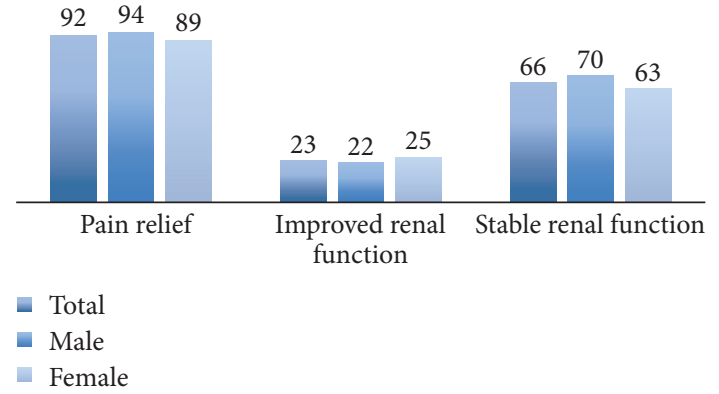

FIgURe 3: Postoperative results.

In contrast, pyeloplasty requires more significant skills by the operator to do the intercorporeal knotting witch prolonged the operation time [8].

A systematic review and meta-analysis of 12 studies by Wang et al. shows that robot-assisted pyeloplasty achieves equivalent results compared to conventionally laparoscopic pyeloplasty, but the robot-assisted procedure has several advantages by shorter suturing-time and shorter length of hospital stay. Furthermore, the robot-assisted procedure has better ergonomics for the surgeon, which improves the success rate of suturing the anastomosis due to more degrees of freedom for movements $[5,9]$.

In 2008, the robot-assisted procedures were introduced in our center with the Da Vinci system, and the numbers of robot-assisted minimal invasive procedures have only increased. The newest report from Sociatas Urologica Denica 2015 shows that $87 \%$ of the pyeloplasty in Denmark are performed with robot assistance [10].

More studies within this field are needed to compare the success rate of our laparoscopic procedures performed traditionally versus robot-assisted procedures. Further studies should be performed under standardized conditions with better measurements of subjective symptoms and objective outcome, simultaneous with a longer follow-up, to evaluate long term outcome.

\section{Conclusion}

Laparoscopic pyeloplasty for UPJO leads to relief of subjective symptoms such as pain in the majority of the patients. Regarding objective outcomes the renal function was mainly preserved or improved. Renal scan drainage showed significant improvement. The success rates are comparable with the results from other centers.

Overall laparoscopic pyeloplasty is an efficient treatment for UPJO.

\section{Disclosure}

An earlier version of this work was presented as a poster at the Congress of the Scandinavian Association of Urology and Urological Nurses, 2013. 


\section{Conflicts of Interest}

The authors declare that there are no conflicts of interest regarding the publication of this paper.

\section{References}

[1] A. E. Tanagho and W. J. McAninch, Smith's General Urologi, Appelton and Lange, Norwalk, Conneticut, USA, 14 edition, 1996.

[2] W. R. Lai, C. A. Stewart, and R. Thomas, "From endopyelotomy to robotic pyeloplasty: what a safari!," Journal of Endourology, 2016.

[3] A. J. Wein, L. R. Kavoussi, A. W. Partin, and G. A. Peters, Campbell-Walsh Urology, Elesevir inc, 11th edition, 2016.

[4] "European Association of Urologi (EAU); Guidelines," https:// uroweb.org/guideline/paediatric-urology/.

[5] F. Wang, Y. Xu, and H. Zhong, "Robot-assisted versus laparoscopic pyeloplasty for patients with ureteropelvic junction obstruction: an updated systematic review and meta-analysis," Scandinavian Journal of Urology, vol. 47, no. 4, pp. 251-264, 2013.

[6] J. Hanske, A. Sanchez, M. Schmid et al., "Comparison of 30-day perioperative outcomes in adults undergoing open versus minimally invasive pyeloplasty for ureteropelvic junction obstruction: analysis of 593 patients in a prospective national database," World Journal of Urology, vol. 33, no. 12, pp. 2107-2113, 2015.

[7] U. Boylu, C. Basatac, T. Turan, F. F. Onol, and E. Gumus, "Comparison of surgical and functional outcomes of minimally invasive and open pyeloplasty," Journal of Laparoendoscopic and Advanced Surgical Techniques, vol. 22, no. 10, pp. 968-971, 2012.

[8] P. Bansal, A. Gupta, R. Mongha et al., "Laparoscopic versus open pyeloplasty: comparison of two surgical approaches- a single centre experience of three years," Indian Journal of Surgery, vol. 73, no. 4, pp. 264-267, 2011.

[9] C. Başataç, U. Boylu, F. F. Önol, and E. Gümüş, "Comparison of surgical and functional outcomes of open, laparoscopic and robotic pyeloplasty for the treatment of ureteropelvic junction obstruction," Turk Uroloji Dergisi, vol. 40, no. 1, pp. 24-30, 2014.

[10] “Sociatas Urologica Denica: Urolap," 2016, http://www.urologi .dk/sites/default/files/nyheder/urolap_200816.pdf. 


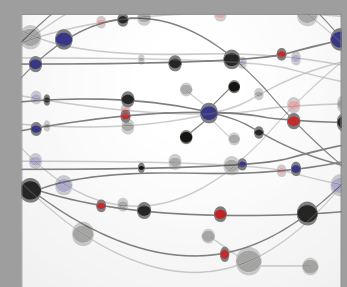

The Scientific World Journal
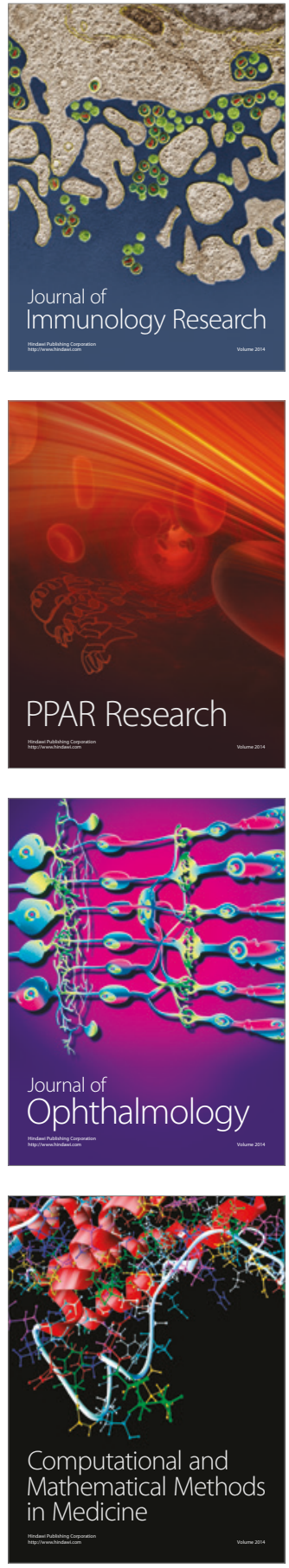

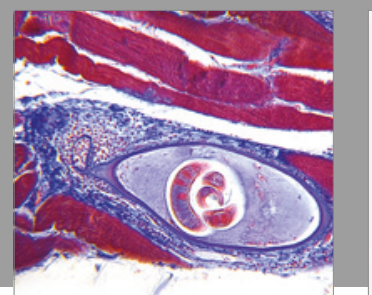

Gastroenterology Research and Practice
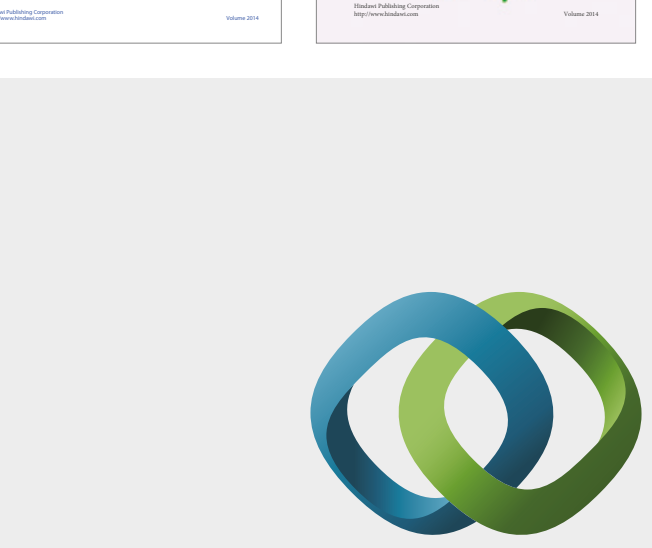

\section{Hindawi}

Submit your manuscripts at

https://www.hindawi.com
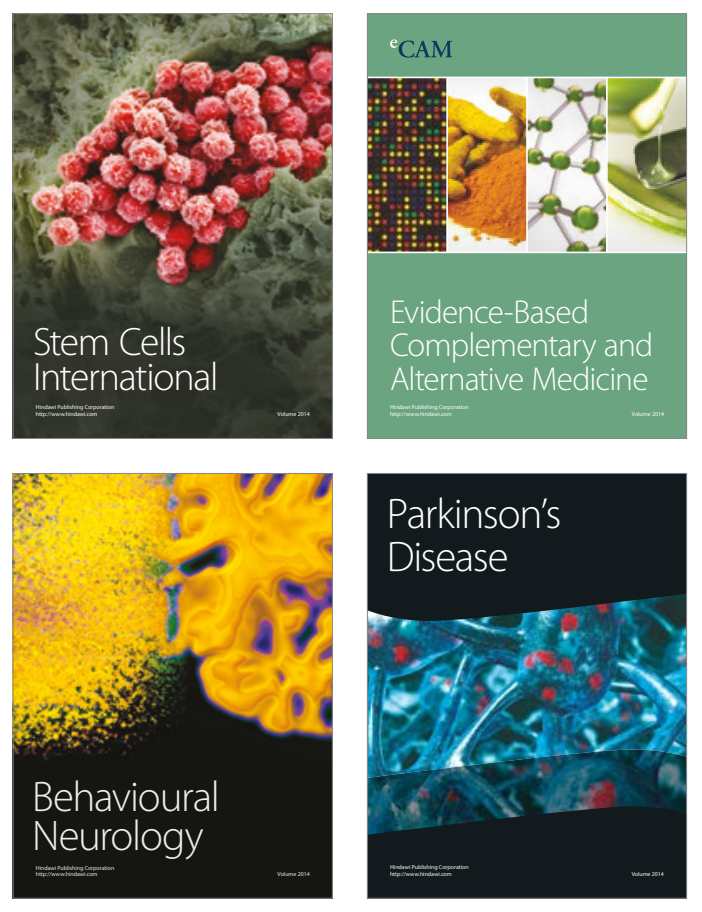
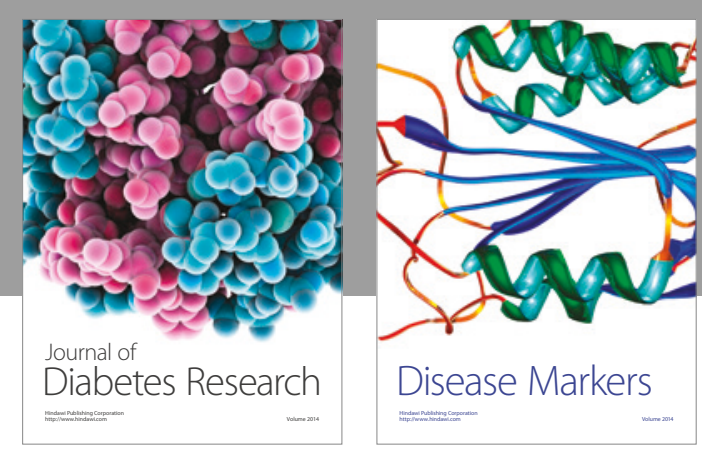

Disease Markers
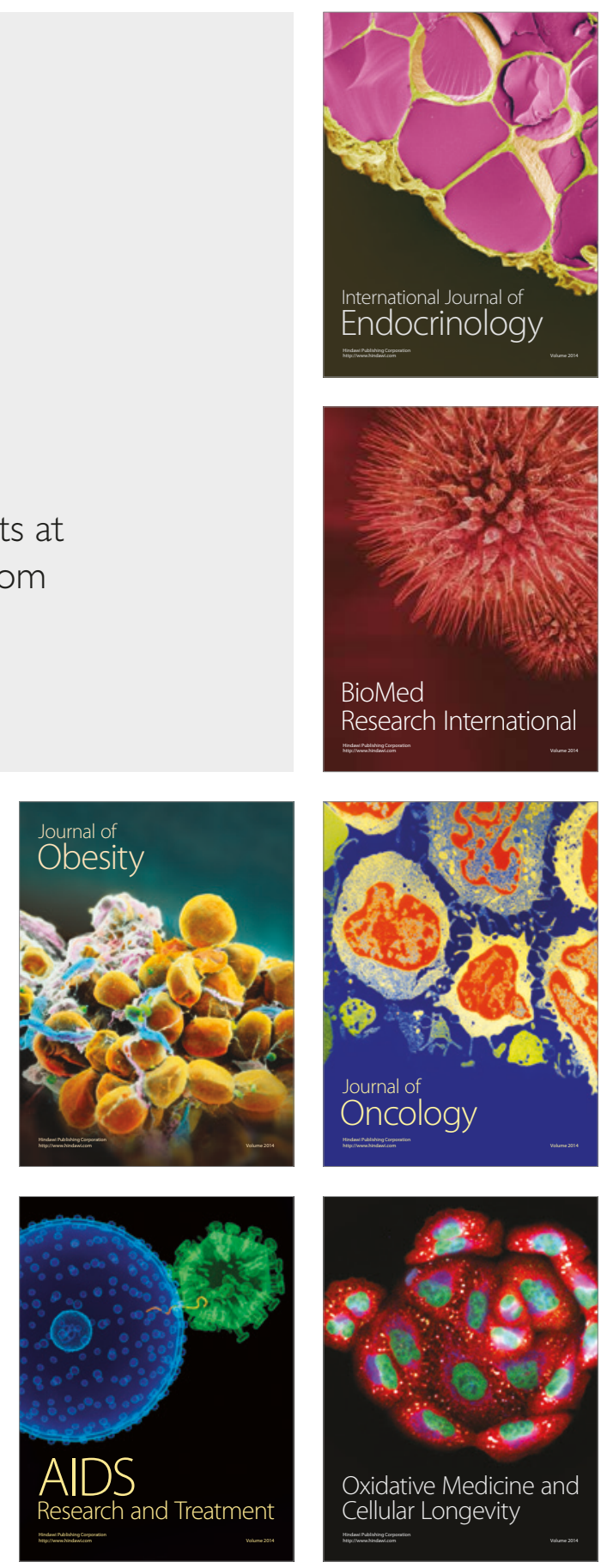